\title{
Apports des TIC dans l'enseignement et l'apprentissage des mathématiques scénarisation pédagogique et pratiques de l'enseignement à distance
}

\section{Contribution of ICT in the teaching and learning of mathematics : pedagogical scripting and practices of distance education}

\author{
Lekbir Chakri ${ }^{1 *}$, My Lhassan Riouch ${ }^{2}$ \\ ${ }^{I}$ Centre régional des métiers de l'éducation et de la formation CRMEF-Rabat, Maroc. \\ ${ }^{2}$ Académie Fès -Meknès, Inspection mathématiques, Direction de Meknès, Maroc.
}

\begin{abstract}
Résumé. L'intégration des TICE (technologie d'information et de communication dans l'enseignement) nécessite une réflexion profonde sur l'évolution des pratiques de classe. Elle nécessite surtout l'innovation dans le choix et dans la conception des situations des apprentissages qui utilisent d'une façon progressive et rationnelle les ressources numériques. Le choix adéquat des approches pédagogiques et didactiques de l'enseignant et des ressources numériques à utiliser ont un impact direct sur l'amélioration de la qualité des apprentissages mathématiques. Dans notre contribution nous réservons une place pour l'impact de la scénarisation et le film éducatif notamment dans l'apprentissage et l'enseignement à distance pour assurer la continuité pédagogique. Nous nous concentrons aussi sur la classe inversée et le e-learning pour leurs rôles dans l'auto-apprentissage des apprenants. Toutefois, il faut signaler que la conception de nouvelles ressources éducatives numériques et leur mise en œuvre présentent un défi majeur pour les enseignants; pour produire une nouvelle ressource numérique, il faut combiner les fonctionnalités de plusieurs logiciels. On parle alors des concepts liés à la genèse et l'orchestration instrumentale $[1,12]$.
\end{abstract}

\begin{abstract}
The integration of ICT (information and communication technology in learning) requires a deep reflection on the evolution of classroom practices. It requires specifically innovation in the choice and planning of pedagogical situations that use digital resources gradually and rationally. The appropriate choice of didactical and pedagogical approaches of the teacher as well as digital resources that would be used have a direct impact on the improvement of the quality of mathematics learning. In our contribution, we reserve a place for the input of scenarisation and
\end{abstract}

* Corresponding author: chakri061@gmail.com 
educational film especially in distance teaching and learning to ensure the continuity of teaching-learning. We also focus on the flipped classroom and e-learning for their roles in the self-learning of learners. However, the conception of digital educational resources and their use is a major challenge for teachers. That is, to produce a new digital resource, the teacher needs to combine functionalities of several software. Here we talk about concepts related to instrumental orchestration and instrumental genesis $[1,12]$.

\section{Introduction}

La plupart des enseignants de mathématiques ainsi que leurs élèves éprouvent énormément de difficultés sur l'accès, l'appropriation, l'adaptation et sur l'utilisation efficace des ressources numériques dans les activités mathématiques. Le but de ce travail est de proposer quelques pistes de remédiation pour surmonter de telles difficultés. Plus précisément, nous nous intéressons aux difficultés majeures au niveau de l'accès et de l'usage des ressources numériques, ainsi que les obstacles qui freinent leurs intégrations dans le processus d'enseignement et d'apprentissage, puis de proposer des solutions convenables. Nous focalisons notre recherche sur le traitement de quelques exemples d'activités ayant recours à plusieurs ressources numériques telles que les logiciels de géométrie dynamique (GeoGebra, Cabi II plus, Casyopée, Math-graphe...). Ces logiciels permettent aussi de modéliser des problèmes réels et de les expérimenter (réalité augmentée) sous différents angles. Dans cet environnement, les enseignants peuvent inviter leurs élèves à se plonger dans un monde virtuel pour acquérir des connaissances et développer des compétences technologiques en parallèle des compétences mathématiques.

Aujourd'hui, personne ne peut nier l'apport considérable des TICE sur l'amélioration de la qualité de l'enseignement et l'apprentissage des différentes disciplines scientifiques, et des mathématiques en particulier. Ainsi, le levier 12 de la réforme de la vision stratégique (20152030) insiste sur le renforcement de l'intégration des technologies éducatives et le développement et l'instauration d'un nouveau modèle pédagogique ouvert, diversifié, novateur dans l'école marocaine. À noter que l'innovation consiste à introduire et à intégrer de nouveaux outils, ressources et supports pédagogiques dans les pratiques de classe. Cependant, l'accès aux ressources, leur appropriation et leur usage efficace en classe posent encore beaucoup de problèmes aux enseignants de mathématiques ainsi qu'à leurs élèves.

La mise en place de la stratégique (2015-2030), vise à accompagner et à soutenir les innovations susceptibles de promouvoir le développement des établissements scolaires (Articles 74-77)i. Le changement des pratiques se réalisera progressivement par l'intégration de supports numériques et d'outils interactifs dans les actes d'enseignement et d'apprentissage, de recherche et de l'innovation. Le changement des pratiques de classe doit commencer par la scénarisation et la planification du déroulement des séances d'apprentissage, la précision des tâches et des rôles de l'enseignant et de l'apprenant et du type d'outils et du matériel pédagogique à utiliser. Les objectifs seront dirigés et focalisés sur l'instauration des pratiques plus constructivistes qui laissent plus d'autonomie aux apprenants en leur favorisant l'interaction et la collaboration pour construire leurs savoirs. Ce changement doit être centré aussi sur la mutualisation, le partage, et les réutilisations des activités et des ressources numériques produites par les enseignants.

L'utilisation des applications comme Geogebra et Cabri à travers la visualisation et le déplacement des objets, tout en gardant les propriétés algébriques et géométriques invariantes, aide les élèves à concevoir un plan de résolution du problème posé. Il est clair que cette visualisation a aussi pour but de motiver et faciliter l'engagement des élèves dans les situations de résolution de problèmes. 
Les outils numériques accompagnent depuis déjà longtemps l'enseignement des mathématiques, et il existe une offre abondante de sites et de ressources gratuites. Mais devant cette profusion, la question de la qualité et de la pertinence des ressources se pose pour les enseignants. Les ressources disponibles manquent de visibilité : disséminées sur des sites web, nationaux et académiques, ou sur des sites associatifs ou personnels, elles restent le plus souvent ignorées des utilisateurs potentiels, car souvent mal décrites pour permettre aux moteurs de recherche généralistes de les retrouver. Une solution à ce problème serait de décrire le contenu des ressources existantes pour en permettre un meilleur référencement et améliorer la possibilité de trouver des ressources pertinentes par des moteurs de recherche. Il s'agit d'associer à chaque ressource des métadonnées comme le type d'activité proposée, le niveau de classe concerné, le thème et le domaine mathématique concerné et encore l'outil numérique mobilisé et un scénario d'usage. Selon Adler [1] le fonctionnement d'une ressource dans et pour les mathématiques à l'école réside dans son utilisation dans un contexte particulier et non dans la simple présence de la ressource.

L'objectif principal de notre travail est d'apporter quelques éléments de réponse aux questions liées à l'accès, l'appropriation des ressources numériques pour rendre leur usage pertinent en vue de l'amélioration de la qualité de l'enseignement et l'apprentissage des mathématiques. Mais aussi de les utiliser dans d'autres approches pédagogiques comme la classe inversée pour favoriser d'autres types d'apprentissages tels que l'investigation et la résolution des problèmes interdisciplinaires et de les partager et les diffuser dans d'autres contextes de formation tels que la formation à distance (E-learning).

\section{Constat - Problématique - Objectifs}

\subsection{Constat sur le terrain}

Notre travail s'inscrit dans le champ des ressources numériques et leurs intégrations dans l'enseignement/apprentissage des mathématiques. Nous nous concentrons sur les ressources numériques pédagogiques (Learning Objects). Nous nous intéressons spécifiquement à celles utilisées dans l'enseignement des mathématiques. Les enseignants de mathématiques et les apprenants au secondaire accèdent et utilisent ces ressources numériques dans leurs enseignements et apprentissage respectivement, mais ceci d'une manière trop restreinte et élaborée. D'après une démarche exploratoire sur un échantillon restreint, nous avons remarqué une méconnaissance des ressources numériques par la plupart des enseignants et un manque d'expertise sur l'utilisation des ressources existantes ainsi que l'absence de volonté d'expérimentation des ressources sur des activités mathématiques. À titre d'exemples :

- Malgré les formations continues sur cabri II plus, la majorité des enseignants ne l'utilise que sur le traçage des figures et non pas sur des activités mathématiques comme la résolution de problèmes.

- Faible maîtrise des logiciels et des ressources numériques mathématiques et difficultés sur l'accès et sur la réutilisation.

- Les contenus des manuels scolaires ne sont pas en cohérence avec les ressources produites et diffusées sur internet.

- Manque de formation concernant la conception, la production, l'expérimentation et l'évaluation des ressources numériques (absence de communauté de pratique). 
$\mathrm{Au}$ Maroc un ensemble de ressources numériques sont accessibles au sein des sites personnels des enseignants innovants en TICE et au sein même du ministère (Taalimtice.ma), mais ce qui est remarquable c'est le manque de description de ces ressources et le scénario d'utilisation. La quantité d'informations sur ces ressources n'est pas suffisante pour être exploitable par les enseignants. De plus, le manque de formation et d'accompagnement des enseignants sur l'accès et sur l'usage de ces ressources rend ces ressources inefficaces. Le rôle des inspecteurs pédagogiques est d'accompagner les projets de formations et de faire des expérimentations dans les classes pour voir l'impact de ces ressources sur l'apprentissage de telle façon à valoriser et à distinguer les ressources qui ont un apport et un intérêt pédagogique et d'éliminer les autres ou penser à les reconstruire pour améliorer leurs qualités. Les enseignants doivent être préparés à collaborer avec les différents spécialistes du milieu éducatif tels que les concepteurs des ressources, les formateurs et les inspecteurs. Par ailleurs, l'intégration des TIC nécessite un travail collaboratif et collectif du corps enseignant pour accentuer la production et rendre l'usage plus pertinent.

Notre constat est basé aussi sur des résultats dégagés de l'analyse d'un questionnaire exploratoire qui a été administré à un échantillon restreint et choisi aléatoirement d'enseignants de mathématiques. Les résultats que nous avons obtenus dans cette exploration montrent que $69,16 \%$ des répondants éprouvent des difficultés d'accès aux ressources numériques et $83,18 \%$ ont des difficultés de leurs usages. De plus le niveau de maîtrise des différentes ressources mathématiques est moyen. A titre d'exemple, il apparait que la majorité des enseignants ne maîtrisent pas les logiciels d'algorithmique et de partage et de production des capsules vidéo, d'ailleurs $72 \%$ ont répondu qu'ils sont des débutant en algorithmique et $87,9 \%$ n'utilisent pas l'algorithmique dans les activités mathématiques, tandis que $68 \%$ des enseignants ont déclaré qu'ils n'ont jamais produit ni d'animations, ni de films éducatifs. En ce qui concerne l'analyse de l'inventaire des trois manuels choisis dans notre recherche concernant le tronc commun scientifique, les propositions des livres sur l'usage des TICE sont extrêmement limitées et ne dépassent guère quelques cas d'intégration de la calculatrice ou du tableur Excel.

\subsection{Problématique}

De ce fait, nous avons jugé important de venir en aide aux enseignants de mathématiques ainsi qu'aux élèves qui éprouvent du mal à trouver les ressources numériques pertinentes et les utiliser ensuite efficacement à des fins pédagogiques. Pour ce faire, nous posons cette problématique sous forme de questions suivantes, auxquelles nous essayons d'apporter quelques éléments de réponse.

1- Quel est l'apport des TICE dans l'amélioration de la qualité des apprentissages des mathématiques?

Dans quelles activités peut-on utiliser et intégrer les ressources numériques?

- Quelle est le rôle de la scénarisation pédagogique dans l'appropriation, l'adaptabilité et l'intégration des ressources numériques?

Quelles sont les obstacles de l'utilisation et l'intégration des ressources numériques dans les pratiques des enseignants de mathématiques? 
Comment rendre les ressources numériques facilement accessibles et

efficacement réutilisables pour les enseignants de mathématiques ainsi qu'aux apprenants?

\subsection{Objectifs de la recherche}

Les objectifs principaux de notre travail se présentent comme suit :

- Valoriser l'apport considérable des TICE sur l'amélioration de la qualité des apprentissages et sur le changement des pratiques de classe.

1- Concevoir des scénarios pédagogiques pour rendre l'usage des ressources pertinent en les intégrant dans des activités telles que l'investigation, la résolution des problèmes ou l'expérimentation en mathématiques.

1- Le film éducatif reste un pilier sur lequel il faut encore travailler pour assurer la continuité pédagogique surtout dans le cadre de l'enseignement à distance dans des contextes tels que celui de l'épidémie du covid-19.

Le schéma suivant montre que la conception d'un scénario pédagogique nécessite une bonne planification pédagogique et didactique de l'activité proposée.

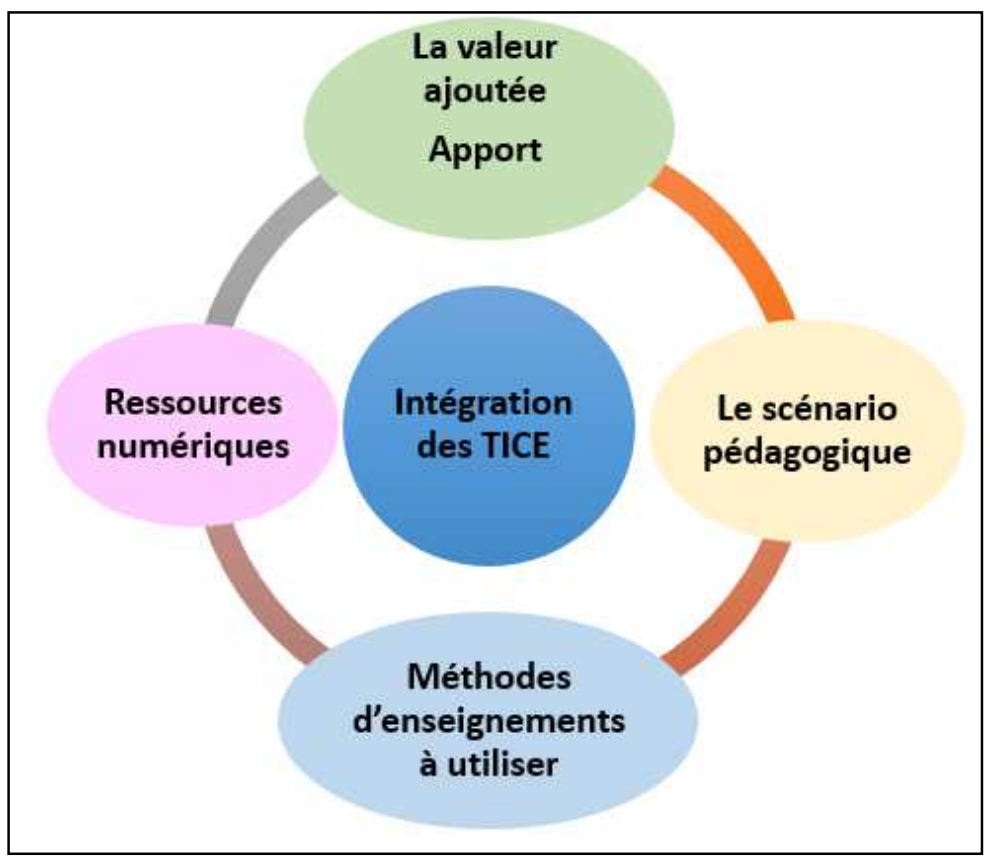

Fig. 1. Planification et intégration des TICE.

Nous présentons une carte conceptuelle qui montre l'apport des TICE : 


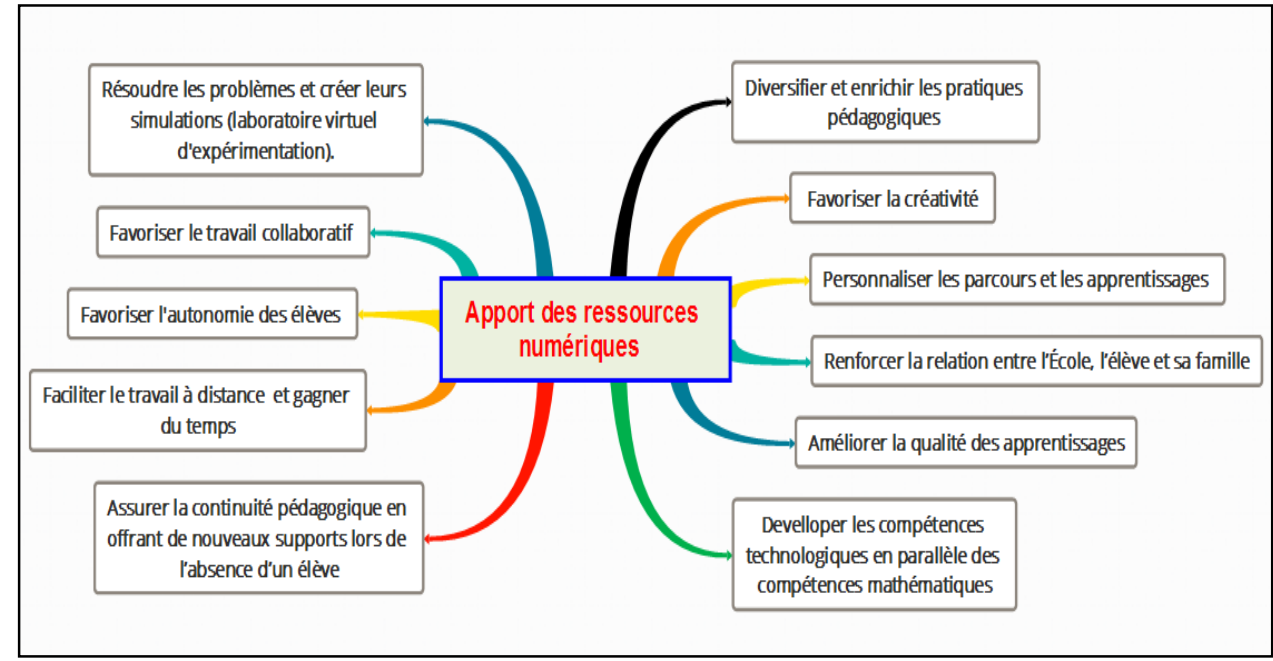

Fig. 2. Apport des ressources numériques.

\section{Propositions de scénarios pédagogiques adéquats pour l'enseignement de quelques concepts mathématiques}

\subsection{Conception des scénarios intégrant les TICE}

Notre travail est orienté vers la conception des situations d'apprentissage visant le développement des compétences mathématiques et technologiques. Notre intention et notre stratégie est de choisir des situations sous forme de problèmes à résoudre et à expérimenter par les TICE. Nous essayons de s'approprier et d'intégrer les ressources numériques dans la résolution. Il est essentiel d'expliquer leurs apports dans l'expérimentation et dans la compréhension du problème (ou phénomène à étudier). Nous allons préciser aussi leur rôle pédagogique dans la motivation des élèves et dans l'instauration du travail collaboratif et expérientiel en classe. Les scénarios proposés organisent les activités de telle façon à s'approprier et intégrer les ressources numériques telles que les logiciels de géométrie dynamique (Geogebra, cabri II plus...), le tableur, les logiciels d'algorithmique (Python, scratch, scrillab...) et le film éducatif dans la résolution. La résolution des problèmes est considérée comme une stratégie que l'enseignant adopte pour développer chez l'apprenant les compétences orientées le plus vers le savoir-faire, vers l'autonomie, la modélisation, la prise de décisions et vers la collaboration. Les objectifs des activités sont orientés vers le développement des compétences du 21 ème siècle telles que la résolution des problèmes liés à la vie courante, la collaboration comme capacité de développer une compréhension partagée, la créativité et l'innovation, la pensée informatique liée à la logique, l'abstraction, la modélisation et à l'intelligence artificielle.

L'instrumentalisation des artefacts (logiciels mathématiques), la conception des ressources, leur appropriation et leur mise en œuvre dans des activités mathématiques ne sont pas des tâches faciles ni pour les enseignants ni pour les élèves. Elles nécessitent plus de recherche au niveau didactique et pédagogique. Le changement de l'environnement de travail, l'adaptation des ressources numériques au programme scolaire nécessitent plus de planification sur le choix et sur le déroulement des activités et plus d'innovation sur la conception des scénarios adéquats. La scénarisation pédagogique a pour objectif de planifier le déroulement de l'activité, d'évaluer sa faisabilité, d'assurer sa cohérence, d'identifier le 
matériel à utiliser et de définir les modalités de travail. Elle permet de retisser des liens interdisciplinaires, en introduisant des contextes concrets d'application des savoirs sur des situations réelles. Le processus de la conception d'un scénario pédagogique se base sur le choix de stratégies, de méthodes et de techniques d'enseignement [2]. Il consiste à préciser les objectifs, les compétences, les rôles, les méthodes, les activités, la durée, les ressources et les outils à utiliser, le produit et enfin la méthode d'évaluation. Le processus nécessite une analyse a priori et une analyse a posteriori de chaque activité proposée.

Le modèle de scénarios que nous proposons donne lieu à un projet, une activité d'apprentissage sous forme d'un problème réel dont la résolution est liée à un domaine mathématique. La modélisation et l'expérimentation technologique se complément arisent pour donner plus de sens aux savoirs mathématiques et de les mobiliser dans la résolution et la production de la simulation. L'activité se fait en deux parties. La première est pratique, elle se fait sur l'environnement écran et en utilisant les ressources numériques pour expérimenter le problème, l'observer pour mieux comprendre son comportement et conjecturer les solutions. La deuxième partie est théorique, et son objectif est de démonter et de valider les solutions par un travail sur papier. Pour concevoir notre graphe de scénario, nous nous sommes inspirés de plusieurs modèles tels que la méthode MISA (Méthode d'ingénierie d'un système d'apprentissage) conçue par Gilbert Paquette (2002) [10].

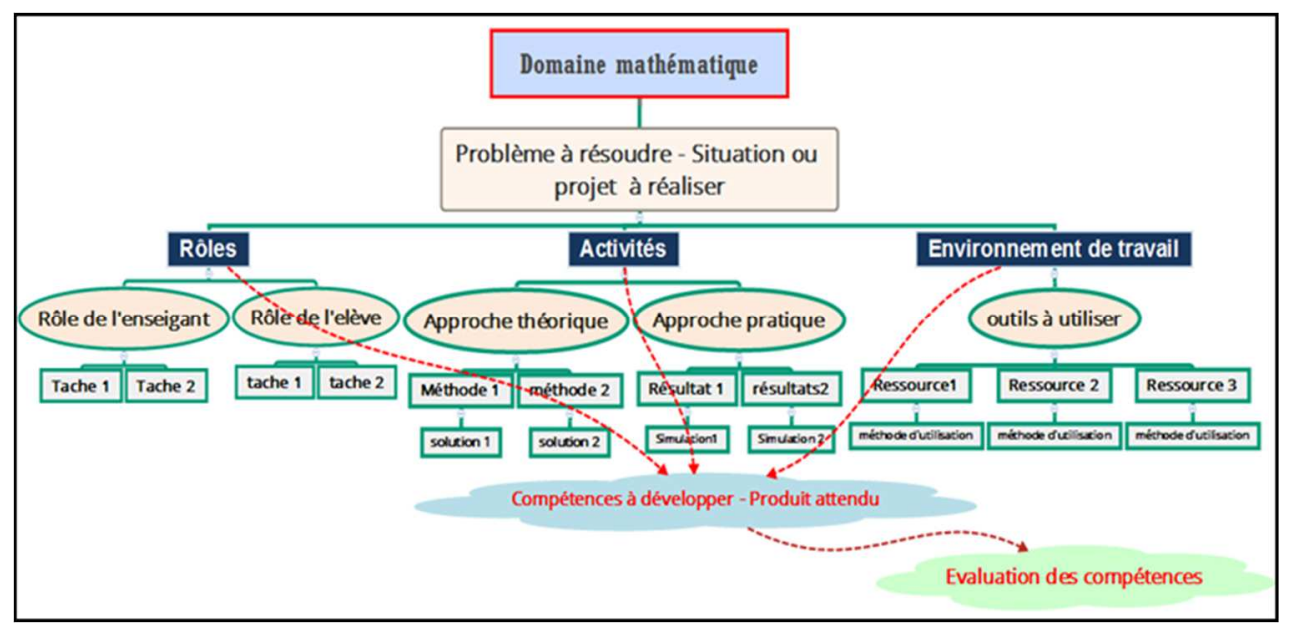

Fig. 3. Processus de scénarisation d'un problème ou situation lié à un domaine mathématique.

\subsection{Inventaire sur les livres: choix des activités intégrant les TICE}

Dans notre recherche, nous avons fait un inventaire sur les manuels scolaires du tronc commun. Nous allons donc diriger notre travail vers les chapitres où nous avons remarqué le manque d'intégration des TICE pour proposer des scénarios dans ce sens. Notre ingénierie didactique consiste à concevoir des mini-projets intégrant les TICE relatifs aux différents domaines mathématiques du niveau tronc commun sciences. Le contenu des activités est choisi et orienté vers un apprentissage significatif qui donne plus de sens aux savoirs mathématiques. Nous insistons aussi sur la réalisation de quelques projets interdisciplinaires et multidisciplinaires et sur la conception de scénarios pédagogiques touchant divers domaines mathématiques (arithmétique, fonctions, résolution d'équations, géométrie dans le plan et dans l'espace) en s'appuyant sur la modélisation et l'expérimentation technologique (simulation). 


\section{Exemples de ressources numériques et situations d'apprentissages intégrant les TICE en mathématiques}

Notre stratégie est de s'approprier, adapter et intégrer les ressources numériques dans les activités mathématiques. La conception des situations d'apprentissage vise le développement des compétences mathématiques ainsi que les compétences technologiques. Elles sont sous forme de problèmes à résoudre et à expérimenter par les TICE (algorithme - simulation, film éducatif).

Dans ce qui suit, nous allons présenter un ensemble de situations intégrant les TICE. Les différentes ressources utilisées sont :

$\checkmark$ Les logiciels de géométrie dynamique (GeoGebra, cabri...),

$\checkmark \quad$ Le tableur (Excel, table sur cabri II plus),

$\checkmark$ Les logiciels d'algorithmique (Python, scratch, ...),

$\checkmark$ Production d'un film éducatif (simulation- récapituler).

\subsection{Exemple 1: Visualisation des abscisses curvilignes sur le cercle trigonométrique}

Les Logiciels de géométrie dynamique permettent de tracer des figures dynamiques, de les modifier librement, de les animer, de les importer et de les exporter librement vers d'autres applications. Ils permettent la construction, la visualisation et l'animation des figures. Nous proposons un exemple concernant la visualisation des abscisses curvilignes dans le cercle trigonométrique niveau tronc commun sciences. (Enroulement de la droite numérique sur le cercle).

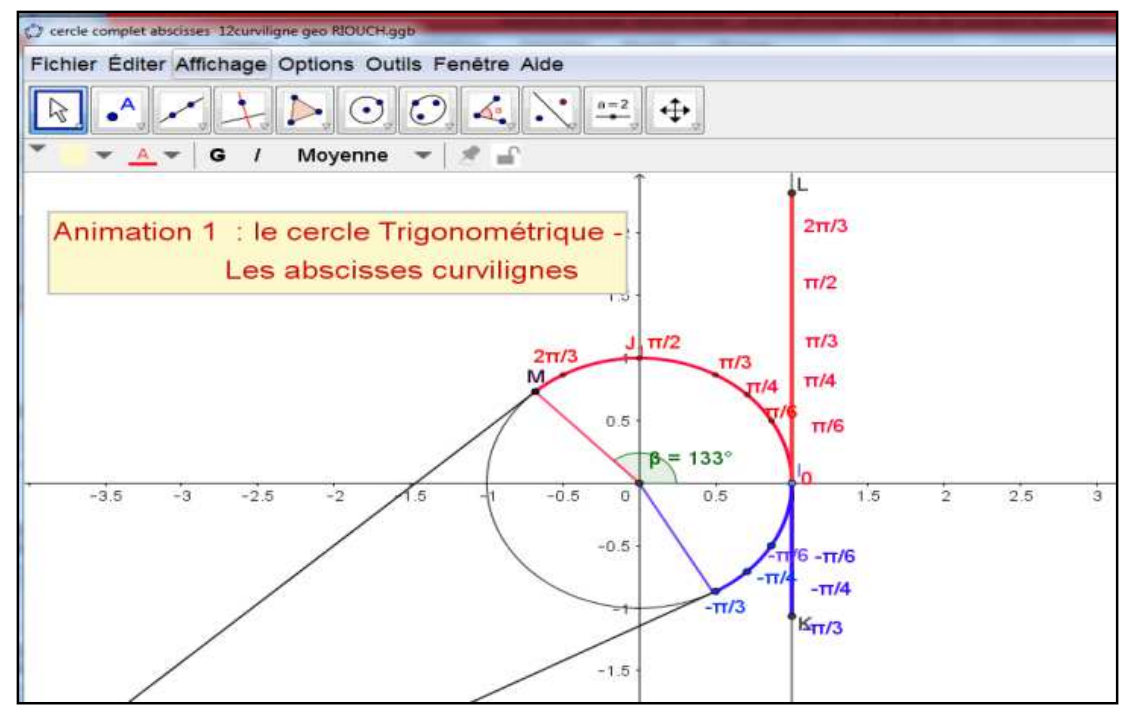

Fig. 4. Présentation avec GeoGebra du cercle trigonométrique (abscisses curvilignes). 


\subsection{Exemple 2 : Les différentes droites sur le triangle}

Les logiciels de géométrie dynamique facilitent le traçage des figures complexes et leurs modifications. Nous présentons ici la droite d'Euler.

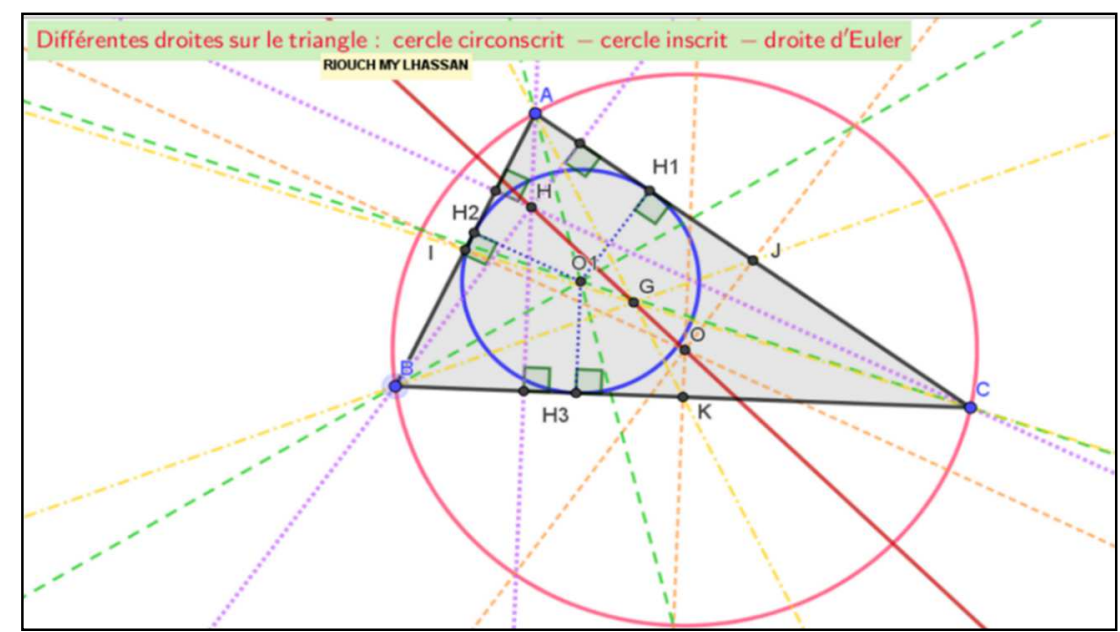

Fig. 5. Cercle circonscrit- inscrit du triangle - centre de gravité- orthocentre - Droite d'Euler.

\subsection{Exemple 3 : Problème d'optimisation}

Dans cet exemple, nous traitons un problème d'optimisation lié au domaine des fonctions numériques. La simulation et l'expérimentation du problème géométrique permettent de transformer la surface en une fonction. Le traçage de la courbe peut ne se faire point par point par le logiciel de géométrie dynamique GeoGebra pour faire émerger la conjecture (le problème à une seule solution qui représente la valeur minimale de la fonction). Ensuite le travail se fera sur papier pour modéliser le problème en transformant l'aire en une fonction trinôme à étudier pour trouver sa valeur minimale.

\section{Enoncé du problème :}

On considère un carré $A B C D$ tel que $A B=4 \mathrm{~cm}$ et $I$ est le milieu du segment $[A, B]$ et $M$ et $N$ deux points libres respectivement sur $[A, D]$ et $[D, C]$ tels que : $A M=D N=x \mathrm{~cm}$.

\section{(Voir figure page suivante)}

1) a) Quelle est la valeur de $x$ pour laquelle la surface $S$ du triangle IMN est égale à $4 \mathrm{~cm}^{2}$ ?

b) Quelle est la valeur de $x$ pour laquelle la surface $S$ du triangle IMN est égale à $5,5 \mathrm{~cm}^{2}$ ?

2) Quelle est la valeur de $x$ pour que la surface du triangle IMN soit minimale? 


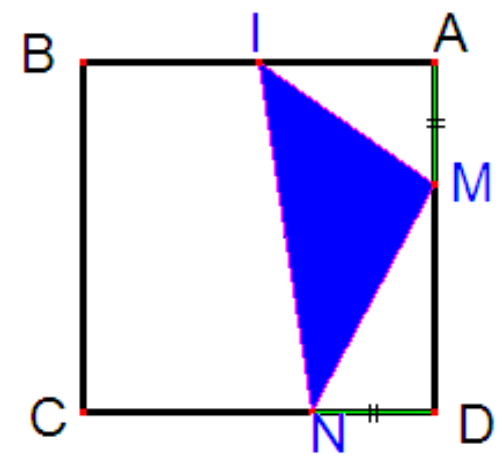

Pour cette situation, nous avons conçu un scénario pédagogique explicitant une analyse a priori de la situation et une analyse de l'activité de l'enseignant en déterminant le rôle et les tâches de chacun d'eux (voir annexe).

La figure suivante est la simulation à produire en utilisant un logiciel de géométrie dynamique.

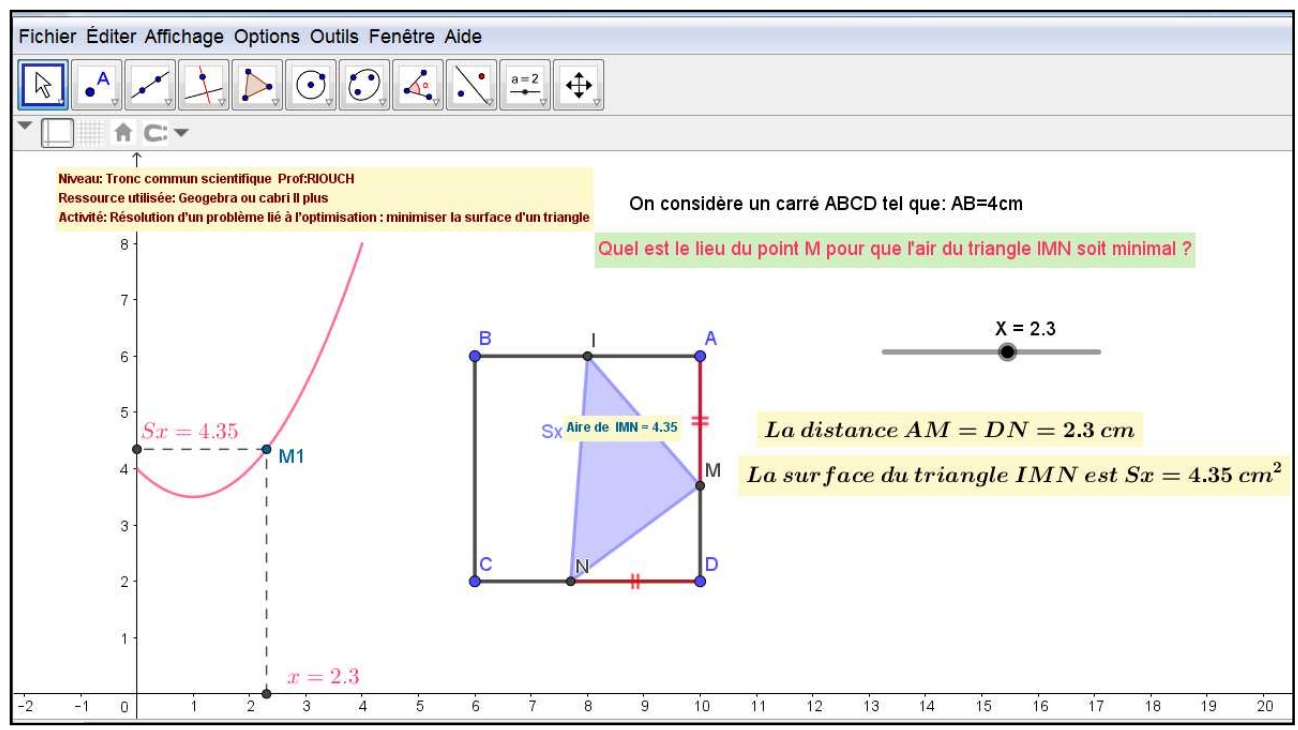

Fig. 6. Simulation du problème d'optimisation avec un logiciel de géométrie dynamique (GeoGebra).

\subsection{Exemple 4 : production d'un film éducatif : démonstration du théorème de Pythagore par diverses méthodes}

On peut produire un film éducatif pour illustrer la démonstration du théorème de Pythagore en combinant les fonctionnalités du logiciel GeoGebra et de Camtasia Studio. 


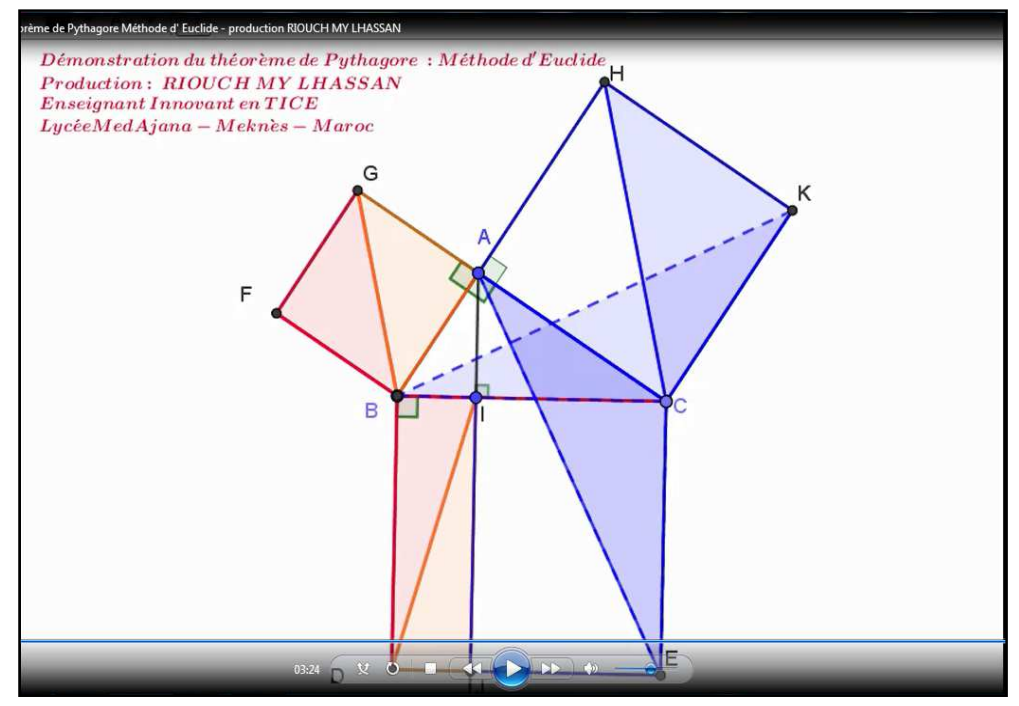

Fig.7. Film éducatif (Démonstration du théorème de Pythagore par la méthode d’Euclide).

\subsection{Exemple 5 : Projet interdisciplinaire Arithmétique- situation problème réelle en physique}

La modélisation mathématique joue un rôle essentiel dans la résolution des problèmes inter et multidisciplinaires. La démarche expérimentale basée sur l'intégration des TICE permet la simulation et la visualisation du problème. Elle favorise la construction progressive des modèles mathématiques qui expliquent le phénomène étudié et qui interprètent son comportement. Elle aide aussi à la formulation, à l'expérimentation et la mathématisation du problème. La visualisation et la manipulation de tous les paramètres du problème facilitent premièrement sa compréhension. Une telle approche développe des compétences transversales et améliore la qualité des apprentissages et donne plus de sens aux savoirs enseignés. Nous allons présenter une situation- problème posée en physique qu'on peut résoudre en mathématiques dans le domaine de l'arithmétique. La création de sa simulation nécessite la collaboration entre l'enseignant des mathématiques et l'enseignant de physiques pour introduire quelques notions et concepts de physique dans la création de sa simulation. Nous utilisons un logiciel de géométrie dynamique, par exemple Geogebra pour créer la simulation.

Énoncé de la situation problème:

Deux mobiles $M_{1}$ et $M_{2}$ partent au départ du point $\mathrm{O}$ et parcourent avec deux vitesses constantes $V_{1}$ et $V_{2}$ deux cercles $\left(C_{1}\right)$ et $\left(C_{2}\right)$ tangent en $\mathrm{O}$.

$M_{1}$ parcourt un tour de $\left(C_{1}\right)$ en $120 s$.

et $M_{2}$ parcourt un tour de $C_{2}$ ) en $252 \mathrm{~s}$.

1. Calculer la durée pour laquelle les deux mobiles $M_{1}$ et $M_{2}$ se rencontrent de nouveau en $\mathrm{O}$ pour la première fois.

2. Déterminer le nombre de tours effectués par $M_{1}$ et $M_{2}$ dans ce cas. 
La création de la simulation de ce problème en physique nécessite d'introduire des notions telles que la période et la vitesse angulaire d'un mouvement circulaire.

Cette activité peut être traitée comme un projet multidisciplinaire dans le cadre des activités des clubs éducatifs de l'établissement. Son objectif est de développer chez les élèves la capacité de mobiliser les TICE pour expérimenter et résoudre un problème réel. L'énoncé peut être reformulé d'une autre façon en physique en introduisant les relations entre vitesse, vitesse angulaire et période et enfin créer la simulation.

Après avoir résolu théoriquement le problème en utilisant le plus petit multiple commun des nombres 120 et 252, qui est $\operatorname{PPCM}(120,2$ 52) $=2520$. Le temps nécessaire serait donc 2520 s. Le mobile $M_{1}$ aurait effectué 21 tours et le mobile $M_{2}$ aurait effectué 10 tours.

On demande aux élèves et en collaboration avec l'enseignant de physique de réaliser un mini- projet en salle d'informatique pour simuler ce problème.

On peut concevoir un protocole de construction pour accompagner les élèves dans la création de la simulation étape par étape sur GeoGebra et aussi sur Excel.

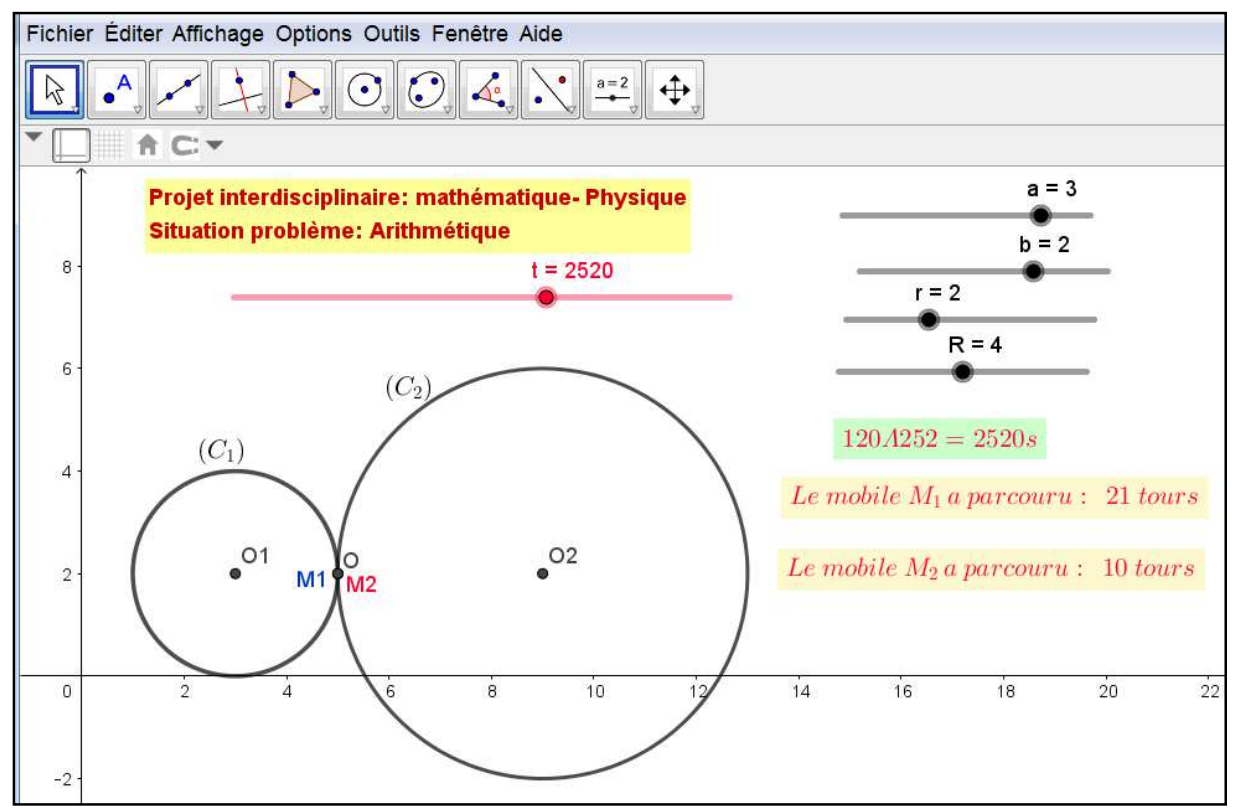

Fig. 8. Simulation du problème en physique par Geogebra.

\section{La formation à distance, le E- Learning et les logiciels de partage et de formation à distance}

Les logiciels de partage de fichiers sont des solutions idéales pour distribuer ou donner accès à distance à des fichiers numériques (documents, présentation, tableur, PDF, vidéo, etc.). L'enseignant peut glisser des documents dans un dossier ou un mur pour les partager et les mettre à la disposition de ses élèves. Les logiciels de partage de fichiers proposent souvent des sauvegardes régulières pour une sécurité et une confidentialité totale. Ils proposent aussi un espace de stockage assez important. Chaque membre disposant des droits d'administration peut accéder aux fichiers autant qu'il le souhaite. Il peut transférer les dossiers, comme il peut aussi les modifier. C'est donc un moyen de partage et de collaboration entre l'enseignant 
et ses élèves. Nous donnons ici l'exemple du logiciel Padlet que nous avons utilisé avec les élèves pour le partage des devoirs et les solutions de quelques examens nationaux.

\subsection{Le logiciel Padlet : un moyen de partage et de travail à distance}

Padlet est un logiciel qui est utilisé pour créer et partager du contenu numérique avec les enseignants et les élèves. C'est aussi un outil utilisé souvent dans la formation à distance. Quelque part entre un document et un créateur de site web à part entière, Padlet permet à chacun de créer le contenu qu'il souhaite, qu'il s'agisse d'un tableau d'affichage rapide, d'un blog ou d'un portfolio. C'est un moyen de partage et de diffusion des ressources numériques. Pour la création d'un compte, des murs et leurs gestions sur Padlet nous présentons la carte suivante :

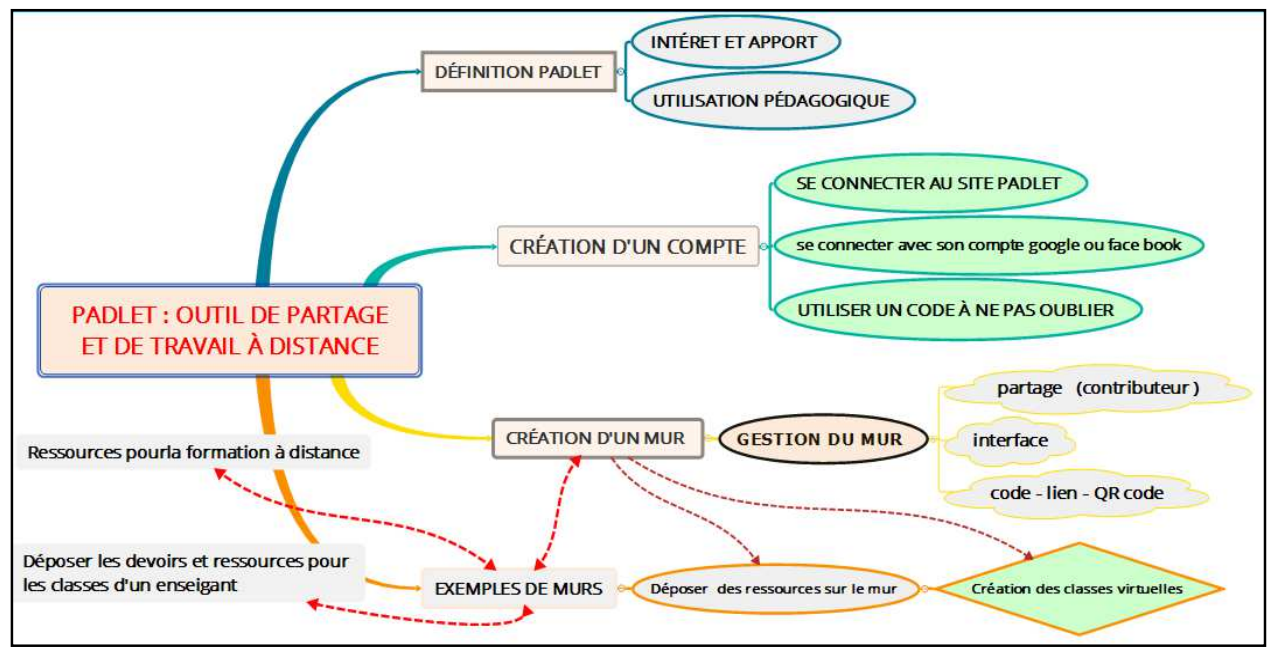

Fig. 9. Carte conceptuelle de la gestion du logiciel Padlet.

Padlet permet de regrouper de nombreuses ressources au même endroit. Elles pourront ainsi être consultées sans qu'il soit nécessaire d'ouvrir plusieurs onglets sur votre navigateur web. Tous les contenus sont visibles en un coup d'œil et regroupés au même endroit sous la forme de post-it épinglés à un mur virtuel. Il suffit ensuite de cliquer sur le post-it pour voir l'intégralité du contenu. On peut donc créer à chaque classe son mur et de travailler et déposer le travail de chaque groupe pour être évalué et partager avec toutes la classe ; c'est en effet un exemple de classe virtuelle. Actuellement, pendant le confinement du covid-19 quelques formateurs l'ont utilisé comme un outil de communication et de partage durant les formations concernant la continuité pédagogique. Le schéma suivant explique la façon de partager l'information, les fichiers de travail et les ressources numériques sur Padlet. 


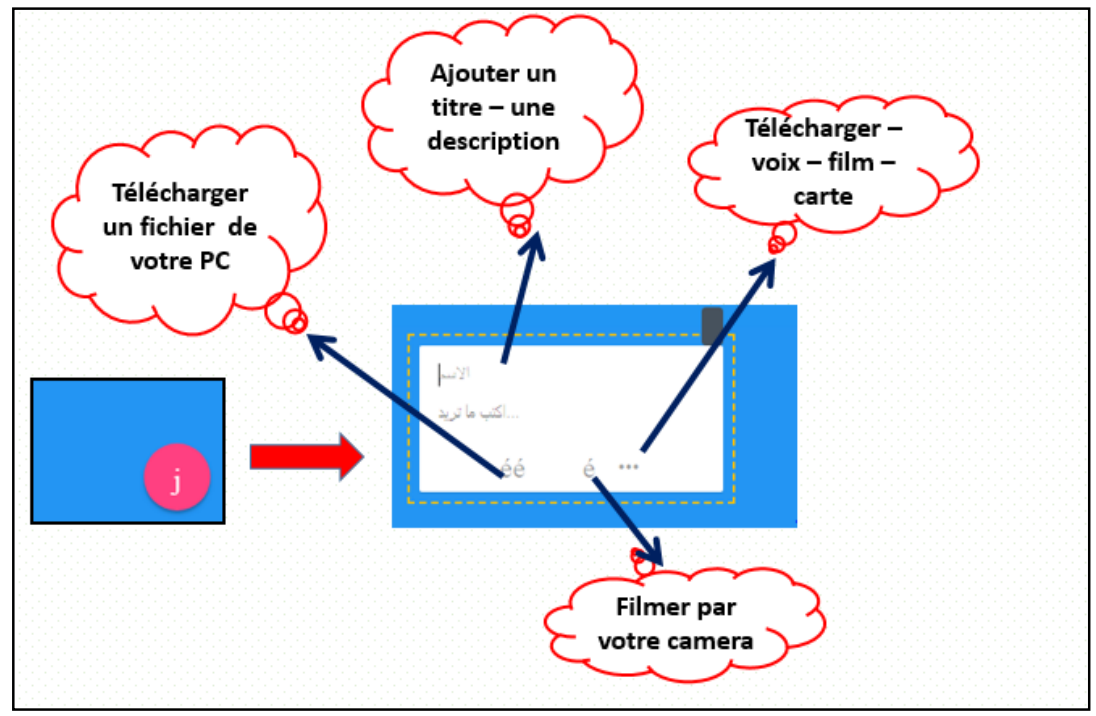

Fig. 10. Padlet outil de travail à distance et de partage des ressources numériques.

\subsection{La classe virtuelle e-learning, la classe inversée}

La classe virtuelle réunit à distance des apprenants et un animateur en même temps et sur une durée définie, de façon synchrone ou asynchrone. C'est une modalité pédagogique différente de l'espace classe avec des objectifs pédagogiques, des activités pédagogiques et des évaluations précises. Elle permet d'accompagner de façon régulière les apprenants pour qu'ils consolident leurs connaissances (savoirs), et développent des habiletés (savoir-faire) et la prise de conscience de leurs capacités individuelles en s'appuyant sur des tutoriels et sur des médias variés tels que l'usage de vidéos. L'enseignement à distance (ou e-learning) prend de plus en plus sa place dans la formation. C'est un moyen d'apprendre, de se former et même de valider des diplômes à distance, c'est-à-dire sans avoir besoin de se rendre dans une école pour assister à des cours. Actuellement les universités offrent des centaines de formations accessibles à distance, à n'importe quel niveau et dans de nombreuses spécialités. Certaines permettent de renforcer la formation continue et la qualification professionnelle des enseignants en leur permettant de réaliser des projets personnels, de partager leurs expériences et d'acquérir et de développer d'autres compétences de travail. Les MOOC (Massive Open Online Courses ou cours en ligne ouverts et massifs) sont en train de révolutionner le paysage de l'enseignement supérieur. Surtout depuis qu'ils permettent la délivrance des certificats... Dans ce type de formation on utilise des plateformes de formation telles que ZOOM, ma classe ; Teams ... pour bien organiser et gérer la formation.

La classe inversée ou « renversée » est un modèle pédagogique qui est apparu ces dernières années. C'est une approche pédagogique qui inverse la nature des activités d'apprentissage en classe et à la maison. Les rôles traditionnels d'apprentissage sont modifiés selon l'expression « les cours à la maison et les devoirs en classe ». L'élève visionne (préalablement à la maison) des vidéos, des cours ou fait des lectures proposées par l'enseignant (ou partagées par d'autres enseignants). En classe, l'élève tente alors d'appliquer les connaissances à la résolution de problèmes et à des travaux pratiques. Le rôle de l'enseignant est alors d'accompagner l'élève dans l'élaboration des tâches complexes. Ce temps dégagé en classe peut être utilisé pour d'autres activités fondées sur l'apprentissage, notamment la pédagogie différenciée et l'apprentissage par projet [8]. 
Dans [9] la classe inversée constitue une hybridation entre l'apprentissage à distance et l'apprentissage présentiel. Dans cette approche l'élève est bien plus autonome. En lui laissant plus de temps pour construire la phase d'apprentissage, l'élève est moins dépendant du contenu transmissif fourni par le professeur. Il ne s'agit plus seulement d'inverser la place de la théorie et de la pratique, mais d'avoir une approche cyclique qui redéfinit les espaces de travail et le découpage des temps.

La classe inversée modifie le rôle traditionnel de l'enseignant. Elle favorise l'enseignement différencié et autonome. En effet, elle permet d'individualiser l'apprentissage et donner plus de temps à chaque apprenant à la maison. Ce qui permet de privilégier le travail collaboratif en classe [5].

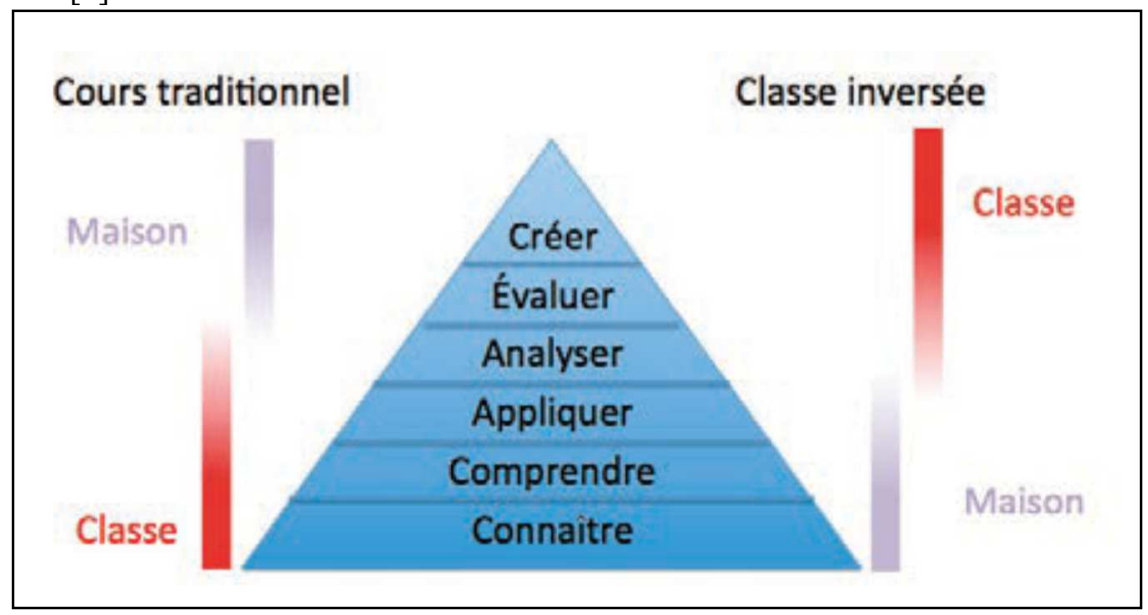

Fig. 11. Activités à la maison et activités en classe.

\section{Conclusion}

Notre travail est orienté vers la conception des situations d'apprentissage visant le développement des compétences mathématiques et technologiques. Notre stratégie est de choisir des situations sous forme de problèmes à résoudre et à expérimenter par les TICE. Nous avons essayé de s'approprier et d'intégrer les ressources numériques dans la résolution de problèmes. Il est essentiel d'expliquer leurs apports dans l'expérimentation et dans la compréhension du problème (ou phénomène à étudier). Les scénarios proposés organisent des activités de résolution de problèmes qui permettent de s'approprier et intégrer les ressources numériques telles que les logiciels de géométrie dynamique (GeoGebra, cabri...), le tableur, les logiciels d'algorithmique (Python, scratch, ...) et le film éducatif. La résolution de problèmes est considérée comme une stratégie que l'enseignant doit adopter pour développer chez l'apprenant les compétences orientées le plus vers le savoir-faire, l'autonomie, la modélisation, la prise de décisions et la collaboration. Les objectifs des activités sont orientés vers le développement des compétences du 21-ème siècle telles que la résolution des problèmes liés à la vie courante, la collaboration comme capacité de développer une compréhension partagée, la créativité et l'innovation, la pensée informatique liée à la logique, à l'abstraction, à la modélisation et l'intelligence artificielle. C'est ce type de situations-problèmes liées à la vie courante et interdisciplinaires qu'il faut envisager d'élaborer et expérimenter par les TICE. 


\section{Références}

1. G. Aldon, Orchestration instrumentale, formation à distance MOOC Efun Math (2017).

2. J. Basque, Texte 441 l'élaboration du scénario pédagogique, http://omafor.technoeducative.com/scenariopedagogique3.pdf

3. M. Ben Henda, Indexation des ressources pédagogiques. https://cel.archivesouvertes.fr/cel-01716681/document.

4. C. Brassard, A. Daele, Un outil réflexif pour concevoir un scénario pédagogique intégrant les TIC. Environnements Informatiques pour l'Apprentissage Humain 2003, Apr. 2003, Strasbourg, France. pp.437-444.

5. H. Dufour, La classe inversée. https://eduscol.education.fr/sti/sites/eduscol.education.fr.sti/files/ressources/techniques/ 6508/6508-193-p44. pdf

6. G. Gueudet, L. Trouche, Ressources en ligne et travail collectif enseignant: accompagner les évolutions de pratique, 2011.

7. Classe Inversée, http://fr.wikipedia.org/wiki/Classe_invers\%C3\%A9e.

8. La notion de Classe Inversée, https://cregut20.fr/wp-content/uploads/2015/05/Lanotion-de-classe-invers\%C3\%A9e.pdf.

9. M. Lebrun, Enseigner et apprendre à l'ère du numérique : entre virage pédagogique et mirage technologique. Conférence. https://www.youtube.com/watch?v=3e AyvzPFmf.

10. Site EDU TIC, Scénario pédagogique la méthode MISA https://edutechwiki.unige.ch/fr/Sc\%C3\%A9nario_p\%C3\%A9dagogique.

11. J. Trgalova, P. R. Richard, Analyse de ressources comme moyen de développement professionnel des enseignants. Acte EMF (espace des mathématiques francophone 2012 GT6, page 911 http://emf.unige.ch/actes-emf-2012/groupe-de-travail-6/

12. L. Trouche, Des artefacts aux instruments, une approche pour guider et intégrer les usages des outils de calcul dans l'enseignement des mathématiques. Actes de l'Université d'été de Saint-Flour. Le calcul sous toutes ses formes. Page 268.

13. Vision stratégique (2015-2030) pilier 12 : Développement d'un modèle pédagogique ouvert, diversifié, performant et novateur, Outils, ressources et supports pédagogiques page (page 43 article 74-77). 
Annexe :

Scénarisation d'une situation-problème liée au domaine des fonctions (optimisation) Exemple : Situation-problème d'optimisation d'une surface (logiciel Geogebra)

\begin{tabular}{|c|c|}
\hline \multicolumn{2}{|c|}{ Scénario pédagogique de la résolution d'un problème géométrique en utilisant } \\
\hline Matière & Mathématiques \\
\hline Public visé & Elèves du tronc commun scientifique \\
\hline $\begin{array}{c}\text { Domaine } \\
\text { mathématique }\end{array}$ & Etude des fonctions \\
\hline Thème & $\begin{array}{l}\text { Optimisation - résolution des équations du 2-ème degré } \\
\text { (Résolution analytique et géométrique) }\end{array}$ \\
\hline $\begin{array}{l}\text { Titre de } \\
\text { l'activité }\end{array}$ & Résolution d'un problème lié à l'optimisation d'une surface \\
\hline Type d'activité & $\begin{array}{l}\text { L'activité se déroule en deux phase une pratique (expérimentale) } \\
\text { se déroule en en salle informatique pour expérimenter le problème } \\
\text { l'autre théorique pour démonter les solutions. Ces deux phases se } \\
\text { complémentarisme pour comprendre, résoudre et expérimenter le } \\
\text { problème }\end{array}$ \\
\hline $\begin{array}{l}\text { Méthode de } \\
\text { travail }\end{array}$ & $\begin{array}{c}\text { Le travail se fera selon les principes de la classe- inversée une } \\
\text { partie libre à la maison et une autre en classe suivi et accompagné } \\
\text { par l'enseignant } \\
\text { La partie théorique : (travail sur papier) } \\
\text { se fera comme un devoir à la maison à rendre sur feuille } \\
\text { La partie pratique (travail sur écran) : } \\
\text { La création de la simulation se fera comme un TP (Travaux } \\
\text { pratiques) sur une salle génie équipé d'ordinateurs et d'un data } \\
\text { show. Le travail se fera en groupe }\end{array}$ \\
\hline \multirow{2}{*}{$\begin{array}{l}\text { Durée de } \\
\text { l'activité }\end{array}$} & $\begin{array}{l}\text { Pour la partie théorique (travail sur papier) elle se fera à la } \\
\text { maison. } \\
\text { (durée ouverte) }\end{array}$ \\
\hline & $\begin{array}{c}\text { La création de la simulation et l'expérimentation du problème le } \\
\text { travail se fera en salle informatique } \\
\text { Durée une heure et demi (1h et3à m) }\end{array}$ \\
\hline Objectifs & $\begin{array}{l}\text { - Résoudre un problème réel en utilisant les savoirs mathématiques } \\
\text { concernant l'étude des fonctions numériques } \\
\text { - Utiliser la démarche expérimentale et de recherche et de preuve } \\
\text { dans la résolution du problème } \\
\text { - Utiliser un logiciel de géométrie dynamique pour créer la } \\
\text { simulation }\end{array}$ \\
\hline & \\
\hline
\end{tabular}




\begin{tabular}{|c|c|c|}
\hline \multirow{4}{*}{$\begin{array}{l}\text { Compétences } \\
\text { à développer }\end{array}$} & \multicolumn{2}{|c|}{ Compétences mathématiques: } \\
\hline & \multicolumn{2}{|c|}{$\begin{array}{l}\text { - } \quad \text { Etre capable de résoudre un problème réel en utilisant les savoirs } \\
\text { et savoirs faire lier aux mathématiques } \\
\text { - } \quad \text { Être capable de modéliser une situation en utilisant les notions } \\
\text { mathématiques. } \\
\text { - } \quad \text { Être capable de résoudre une équation de } 2 \text {-ème degré } \\
\text { - } \quad \text { Être capable d'étudier une fonction numérique et de dresser son } \\
\text { tableau des variations } \\
\text { - } \quad \text { Etre capable de déterminer les extrémums (valeur maximale ou } \\
\text { minimale) d'une fonction. }\end{array}$} \\
\hline & \multicolumn{2}{|c|}{ Compétences technologiques: } \\
\hline & \multicolumn{2}{|c|}{$\begin{array}{l}\text { - Etre capable d'utiliser les commandes principales d'un logiciel } \\
\text { de géométrie dynamique } \\
\text { - Être capable de crée une figure dynamique } \\
\text { - Être capable de créer la simulation d'un problème } \\
\text { - Être capable d'expérimenter, de faire des essais, d'émettre une } \\
\text { conjecture, de la tester, de la « consolider » ou l'invalider }\end{array}$} \\
\hline \multirow{2}{*}{$\begin{array}{l}\text { Etapes de } \\
\text { déroulement de } \\
\text { l'activité }\end{array}$} & $\begin{array}{l}\text { Partie Pratique : } \\
\text { en classe ou en salle Génie }\end{array}$ & $\begin{array}{l}\text { Partie théorique : } \\
\text { à la maison }\end{array}$ \\
\hline & $\begin{array}{l}\text { - Création de la simulation du } \\
\text { problème } \\
\text { - } \quad \text { Experimenter les deux méthodes }\end{array}$ & $\begin{array}{l}\text { - Résoudre le problème } \\
\text { en le modélisant à } \\
\text { l'aide d'une fonction } \\
\text { trinôme à étudier }\end{array}$ \\
\hline $\begin{array}{l}\text { Outils ou } \\
\text { ressources à } \\
\text { utiliser }\end{array}$ & \multicolumn{2}{|c|}{$\begin{array}{l}\text { Une salle équipée d'ordinateurs, un data show, un logiciel de } \\
\text { géométrie dynamique tel que : Geogebra- cabri- Math graphe et } \\
\text { logiciel Camtasia pour transformer la simulation en film. }\end{array}$} \\
\hline $\begin{array}{c}\text { Prérequis } \\
\text { mathématiques }\end{array}$ & \multicolumn{2}{|c|}{$\begin{array}{ll}\text { - } & \text { Savoir Calculer l'aire d'une surface } \\
\text { - } & \text { Savoir étudier les variations d'une fonction } \\
\text { - } & \text { Savoir déterminer extrémums d'une fonction }\end{array}$} \\
\hline $\begin{array}{l}\text { Pré-requis } \\
\text { technologiques }\end{array}$ & \multicolumn{2}{|c|}{$\begin{array}{ll}\text { - } & \text { Savoir utiliser les commandes principales du logiciel } \\
\text { - } & \text { Geogebra (traçage des points libre- liés par coordonnés) } \\
\text { - } & \text { Savoir utiliser un curseur } \\
\text { - } & \text { Savoir afficher les valeurs de chaque variable } \\
\text { - } & \text { Savoir utiliser la commande trace } \\
\end{array}$} \\
\hline
\end{tabular}

$\underline{\text { Rôles de l'enseignant et de l'élève }}$ 


\begin{tabular}{|c|c|}
\hline Rôle de l'enseignant: & Rôle de l'élève: \\
\hline $\begin{array}{l}\text { - Préparer tous les fichiers de l'activité et } \\
\text { la fiche de l'élève } \\
\checkmark \text { Enoncé } \\
\checkmark \text { Etapes de la création de la } \\
\text { simulation } \\
\text { - Présenter et expliquer les étapes de } \\
\text { l'activité (objectifs- méthode de } \\
\text { travail) } \\
\text { Préparer le Protocole de construction } \\
\text { de la figure par le logiciel choisi. } \\
\text { - Suivre le travail de chaque groupe } \\
\text { concernant les deux parties théoriques } \\
\text { et pratiques } \\
\text { Aider les groupes qui ont des } \\
\text { problèmes les élèves dans } \\
\text { Aider les la validation } \\
\text { l'institutionnalisation et la } \\
\text { des solutions }\end{array}$ & 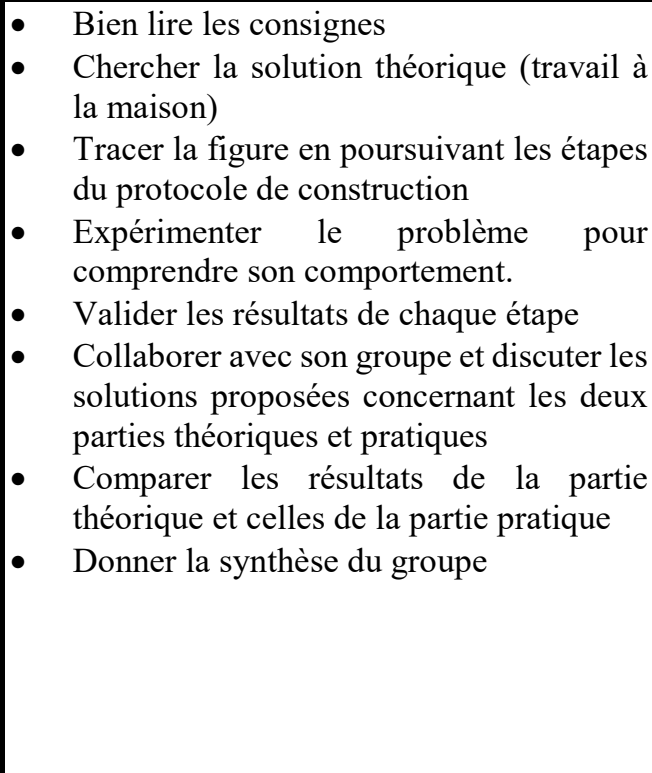 \\
\hline
\end{tabular}

